\title{
Entwicklungen und Herausforderungen der Zukunft
}

Kryptowährungen haben grosse Zukunftschancen. Sie bieten eine Alternative zum Bargeld, insbesondere für Menschen, die ihre Privatsphäre gegenüber staatlichem Zugriff wahren wollen (Wohlmann, 2020). Damit locken sie allerdings auch zwielichtige Machenschaften an, die über Kryptowährungen abgewickelt werden. Hier wäre eine vorsichtige, die Chancen wahrende, Regulierung durch die Staaten sinnvoll. Dies gilt auch für die Bilanzierung, die mit dem weiteren Vordringen der Kryptowährungen in die Realwirtschaft zur Nutzung als Zahlungsmittel immer mehr Unternehmen betreffen wird. Eindeutige Regeln sind wünschenswert. Sie sind sicherlich auch leicht einzuführen, da sich die Rechnungslegungsstandards im DACH-Raum inklusive der IFRS nur geringfügig unterscheiden. Insofern erscheint eine international vergleichbare Regelung zur Förderung der Transparenz sinnvoll und machbar.

Neue Entwicklungen wie Stablecoins und von Zentralbanken emittierte Kryptowährungen können die Entwicklung von Kryptowährungen nachhaltig unterstützen, da sie das dem System innewohnende Vertrauensproblem lösen können.

Durch die Globalisierung wächst die Menge an grenzüberschreitenden Zahlungen. Diese dauern lange und die Transaktionskosten sind hoch, was insbesondere die Einbindung von Entwicklungsländern in die globalisierte Volkswirtschaft behindert. Mit Kryptowährungen würde sich dieses Problem lösen lassen, insbesondere weil sie auch am etablierten Bankwesen vorbei gehandelt werden können. Dies eröffnet die Möglichkeit für alle, die über ein digitales Endgerät verfügen, am Welthandel teilzunehmen, ohne dazu Zugang zu einem etablierten Bankensystem zu haben. Damit kann ein ungeheurer Entwicklungsschub durch Kryptowährungen entstehen. Voraussetzung dafür ist allerdings, dass die Menschen dem System vertrauen und das Problem der illegalen Transaktionen durch sinnvolle und vernünftige Regulierung gelöst wird.

(C) Der/die Autor(en), exklusiv lizenziert durch Springer Fachmedien

Wiesbaden GmbH, ein Teil von Springer Nature 2021

S. Behringer et al., Kryptowährungen im Rechnungswesen, essentials, https://doi.org/10.1007/978-3-658-36054-2_5 